\title{
Clinical Significance of Choroidal Thickness in Eyes with Ocular Ischemic Syndrome
}

\author{
Dae Hwan Kwon, Yu Cheol Kim, Kyung Tae Kang \\ Department of Ophthalmology, Keimyung University Dongsan Hospital, Keimyung University School of Medicine, Daegu, Korea
}

Purpose: To determine the clinical significance of choroidal thickness and evaluate changes in choroidal thickness over time in eyes affected by ocular ischemic syndrome (OIS).

Methods: Medical records of 16 patients diagnosed with OIS between November 2017 and August 2019 were retrospectively reviewed. In every nine areas of the Early Treatment Diabetic Retinopathy Study grid, we compared the differences in choroidal thickness between the eyes with OIS and unaffected eyes, and its change in the OIS eyes during the follow-up period using swept-source optical coherence tomography. We analyzed the best-corrected visual acuity, intraocular pressure, fluorescein angiography (FAG) parameters, systemic diseases, and the duration of symptoms. Correlation between FAG parameters and the choroidal thickness value ratio in the OIS eyes and the unaffected eyes, and changes in the choroidal thickness in the OIS eyes during follow-up were investigated.

Results: Median age of the subjects was 67.5 years. In the OIS eyes, all FAG parameters were delayed at the initial examination. Best-corrected visual acuity and intraocular pressure were significantly different between the OIS and unaffected eyes. While the choroidal thickness in every nine areas of Early Treatment Diabetic Retinopathy Study in the OIS eyes was lesser than the unaffected eyes, the choroidal thickness of inner temporal, outer nasal, and outer temporal area showed statistically significant difference. During follow-up, changes in choroidal thickness of OIS eyes as well as correlation between the FAG parameters and the choroidal thickness value ratio between the OIS eyes and the unaffected eyes were not found to be statistically significant.

Conclusions: The choroidal thickness of the eyes with OIS was significantly less compared with the unaffected eyes. We inferred that choroidal thinning takes place in the early phase of the disease as the changes in choroidal thickness during the follow-up period were not significant.

Key Words: Choroid, Ischemia, Optical coherence tomography, Retina

Received: September 30, 2021 Final revision: November 16, 2021 Accepted: November 24, 2021

Corresponding Author: Kyung Tae Kang, MD, PhD. Department of Ophthalmology, Keimyung University Dongsan Hospital, Keimyung University School of Medicine, 1035 Dalgubeol-daero, Dalseo-gu, Daegu 42601, Korea. Tel: 82-53-258-7858, Fax: 82-53-258-4558, E-mail: kkt0604@dsmc. or.kr
Ocular ischemic syndrome (OIS) is caused by hypoperfusion of the eye due to occlusion or stenosis of the common and/or internal carotid arteries [1]. OIS presents with a variety of symptoms including visual loss, changes in the visual field, and ocular pain. These may lead to persistent blindness accompanied by secondary changes [2]. Every year, the number of new diagnosed cases of OIS are 7.5 per 
million population [3]. The incidence rate increases with age, which seems to be related to the cause of OIS. The 5 -year mortality rate is approximately $40 \%$, and OIS is sometimes the first manifestation of a fatal carotid artery disease $[4,5]$.

Occlusion or stenosis of the carotid artery can also influence choroidal blood flow and thickness. The choroid is a critical layer in the ocular structure and assessing changes in its thickness in association with specific diseases is of increasing importance in clinical research [6,7]. Fortunately, we can easily carry out non-invasive measurements of choroidal thickness by enhanced depth imaging optical coherence tomography (OCT) [8]. Despite this, there are very limited studies on choroidal thickness in eyes with OIS. Kang et al. [9] reported that 15 patients showed decreased choroidal thickness in the OIS affected eyes compared to the unaffected eyes, and choroidal thinning represents impaired choroidal circulation in these patients. Kim et al. [10] also found that the choroidal thickness and eye volume were lower in 19 patients with OIS compared to those with unaffected eyes. In the present study, we analyzed the differences in choroidal thickness between the OIS affected and unaffected eyes and the changes in choroidal thickness during follow-up. We also evaluated the correlation between choroidal thickness of OIS affected eyes and their fluorescein angiography (FAG) parameters. Through this, we sought to identify the significance of choroidal thickness in the clinical course of OIS.

\section{Materials and Methods}

\section{Study design and participants}

We retrospectively reviewed the medical records of 16 patients diagnosed with OIS at Keimyung University Dongsan Hospital in South Korea between November 2017 and August 2019. Confirmed cases of unilateral OIS were included in the study. OIS diagnosis was based on typical clinical symptoms, slit-lamp biomicroscopy, FAG, brain magnetic resonance angiography (MRA) or carotid Doppler examination to evaluate carotid artery stenosis or occlusion. Moreover, even patients with unclear stenosis on these carotid imaging tests were considered OIS patients if they had clear ophthalmic FAG findings, two or more of the following: (1) delayed patchy choroidal filling, (2) in- creased retinal arteriovenous transit time, and (3) late retinal artery staining. Patients were excluded from the analysis if they had bilateral OIS, intraocular infection/ inflammation, proliferative diabetic retinopathy, or history of intraocular surgery (except uneventful cataract surgery). Cases with inadequate follow-up data, including best-corrected visual acuity (BCVA), intraocular pressure (IOP), swept-source (SS)-OCT and FAG, were also excluded from the study.

The obtained medical records were reviewed for the following: age, sex, medical conditions including hypertension, diabetes, and dyslipidemia; history of cataract surgery or the presence of neovascular glaucoma; duration of the symptoms; and data of ophthalmic examination.

This study was approved by the institutional review board of Keimyung University Dongsan Hospital (No. 2021-04-026) and was conducted in accordance with the Declaration of Helsinki. The requirement for written informed consent was waived because the study was retrospective.

\section{Ophthalmic examinations}

All patients underwent comprehensive ophthalmic examination, inclusive of BCVA as the logarithm of the minimum angle of resolution (logMAR) scale, refraction, IOP using Goldmann applanation tonometry, slit-lamp biomicroscopy, fundus examination, SSOCT, and FAG using a Heidelberg HRA (Heidelberg Engineering, Heidelberg, Germany). The FAG parameters included arm-to-choroid circulation time (ACT), arm-to-retina circulation time (ART), and arteriovenous transit time (AVT). ACT was measured as the length of time from the initiation of fluorescein dye injection to the first filling within the choroid. ART was defined as the length of time between the initiation of injection and the first indication of fluorescence within the retinal arteries. AVT was measured from the first appearance of staining with the retinal arteries until the retinal veins were completely filled. OCT examinations were performed using SS-OCT (DRI Triton; Topcon, Tokyo, Japan). According to the protocol of the SS-OCT system, we obtained 64 to 128 averaged horizontal scan images in a $12-\mathrm{mm}$ transverse scan range for each eye. The 128 single images were averaged using embedded software to 
improve the quality. The lateral and in-depth tissue resolution were 20 and $8 \mu \mathrm{m}$, respectively. Subfoveal choroidal thickness was defined as the distance between the outer border of the hyperreflective line representing the retinal pigment epithelium and the choroidoscleral interface. The automated built-in calibration software in the IMAGEnet 6 viewer (Topcon) was used to determine the distance between these lines with the provide ruler. The choroidal thickness (including subfoveal choroidal thickness) in each Early Treatment Diabetic Retinopathy Study (ETDRS) area was measured using the vertical distance on the OCT images from the retinal pigment epithelium layer to the choroidoscleral interface by one observer's constant method. The study was conducted by a retina specialist (KTK). The SS-OCT also provided the average retinal thickness of the center and eight quadrants according to the ETDRS grid (center, inner/outer superior, inner/outer inferior, inner/outer nasal, and inner/outer temporal) in all cases (Fig. 1A, 1B).

\section{Statistical analyses}

The comparison of variable parameters between OIS eyes and unaffected eyes and changes in choroidal thickness in eyes with OIS during follow-up were assessed using the Wilcoxon signed-rank test. Spearman's rank correlation coefficient was used to evaluate the correlation between choroidal thickness value ratio (between the OIS eyes and the unaffected eyes) and FAG parameters (Fig. 2). It was also used to analyze the correlation between the change in choroidal thickness in the OIS eyes during follow-up and FAG parameters. Statistical significance was set at $p<0.05$. Statistical analysis was performed using IBM SPSS ver. 25.0 (IBM Corp., Armonk, NY, USA).

\section{Results}

\section{Patient and ophthalmic characteristics}

A total of 16 patients were included in this study. The baseline characteristics of these patients are shown in Table 1 . The median age was 67.5 years and 15 patients were
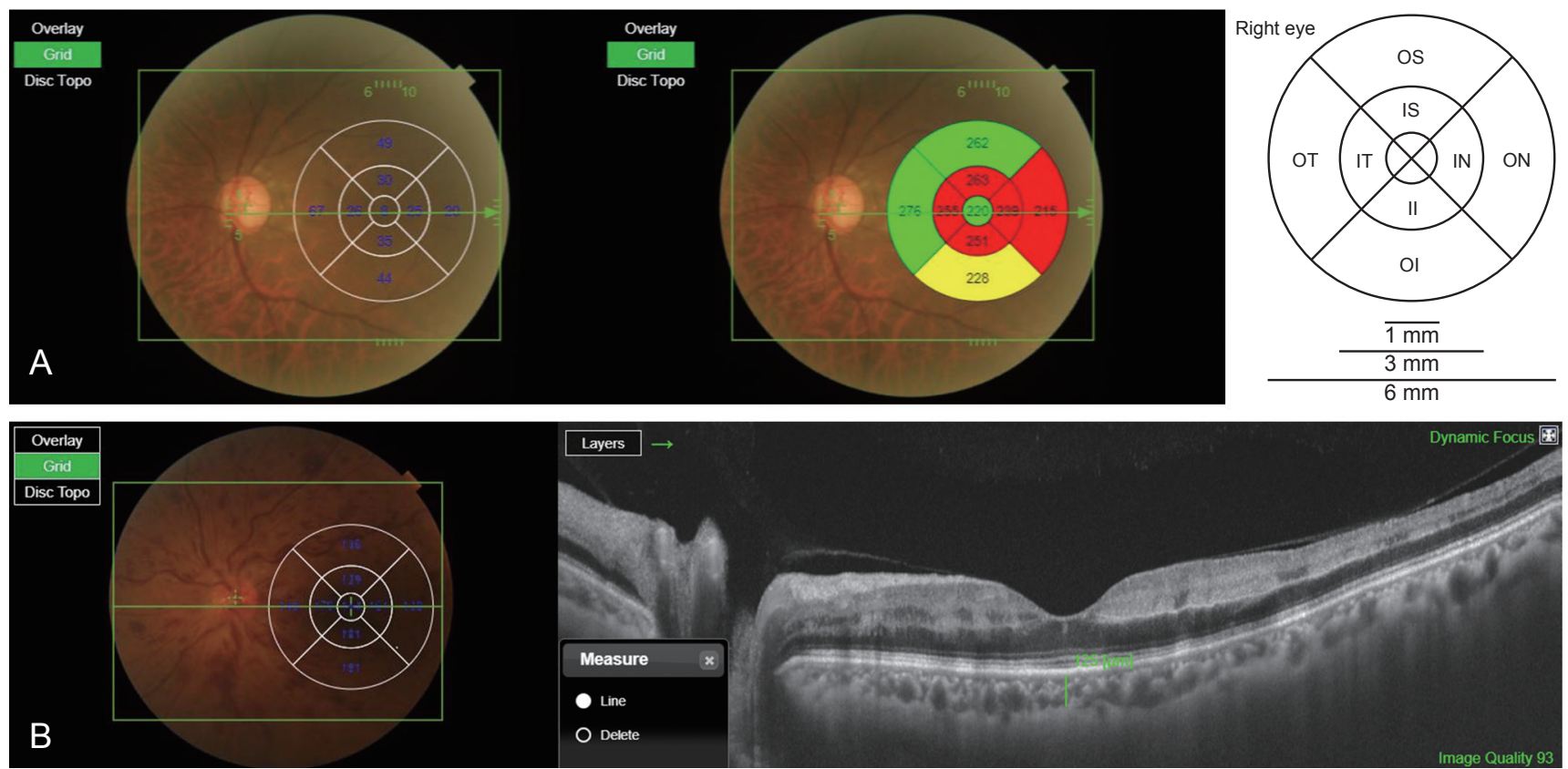

Fig. 1. Measurements of choroidal and retinal thickness. The swept-source optical coherence tomography provided (A) the average retinal and (B) choroidal thickness of center and eight quadrants according to the Early Treatment Diabetic Retinopathy Study grid. (B) After resetting the B-scan by placing the cursor in the center of each area, the vertical distance from the layer of retinal pigment epithelium to the choroid-scleral boundary was automatically measured using the line function. $\mathrm{C}=$ central; II $=$ inner inferior; IN $=$ inner nasal; IS $=$ inner superior; IT = inner temporal; $\mathrm{OI}=$ outer inferior; $\mathrm{ON}=$ outer nasal; $\mathrm{OS}=$ outer superior; $\mathrm{OT}=$ outer temporal. 


\begin{tabular}{|c|c|c|c|c|c|c|c|c|c|c|c|}
\hline $\begin{array}{l}0 \\
0 \\
0 \\
000 \\
0 \\
0\end{array}$ & $\begin{array}{c}0 \\
0^{\circ} \\
90 \\
800 \\
0\end{array}$ & 0 & $\begin{array}{l}0 \\
0^{\circ} \\
\infty \\
\infty \infty^{\infty} \infty \\
0\end{array}$ & $\log _{0}^{\circ} \infty^{\infty} 0$ & $\begin{array}{l}0 \\
0^{\circ} 0 \\
0 \\
08^{\circ} \circ 0\end{array}$ & $\begin{array}{c}0 \\
80 \\
000 \\
0 \\
0 \\
0\end{array}$ & $\begin{array}{ll}0 \\
8 & 0 \\
0 & 0\end{array}$ & 0 & $\begin{array}{l}0 \\
0 \\
0 \\
0 \\
0 \\
0 \\
0 \\
0\end{array}$ & $\begin{array}{c}0 \\
0 \\
\circ 800 \\
080 \\
8\end{array}$ & 0 \\
\hline $\begin{array}{l}0 \\
00880 \\
00 \\
0\end{array}$ & $\begin{array}{l}\circ \\
88 \circ 0 \\
0\end{array}$ & 0 & $\begin{array}{c}0 \\
0 \\
0 \\
0\end{array}$ & $\begin{array}{l}\circ \\
0 \\
\circ 88^{\circ} \\
0 \\
0\end{array}$ & $\begin{array}{l}0_{0} \\
8_{0}^{\circ} \infty 0_{0}\end{array}$ & $\begin{array}{c}0_{0} \\
0_{0}^{\infty} 0^{\circ} 0^{\circ}\end{array}$ & $\begin{array}{ll}8 & 0 \\
808 & \\
0 & 0\end{array}$ & 0 & $\begin{array}{l}0 \\
0 \\
088 \\
0\end{array}$ & $\begin{array}{r}8 \\
0888 \\
0\end{array}$ & 0 \\
\hline $\begin{array}{lll}0 & \\
0 & & \\
0 & 0 \\
08 & 8 & 0 \\
0 & 0\end{array}$ & $\begin{array}{l}0 \\
80 \\
88^{\circ} \circ \\
0\end{array}$ & 0 & $\begin{array}{l}0 \\
0_{0}^{\infty} \\
\infty_{0}^{\infty} \infty \infty_{0} \\
0^{\circ}\end{array}$ & $\begin{array}{l}8^{\circ} \\
8_{0}^{\circ} \circ \infty^{\circ} \circ\end{array}$ & $\begin{array}{l}0 \\
80^{\circ} \\
8000 \\
800\end{array}$ & $\begin{array}{c}0 \\
0 \\
0.0 \\
0000 \\
0.08^{\circ}\end{array}$ & $8^{\circ} 8^{\circ}$ & 0 & 0.80 & $\begin{array}{c}0 \\
\circ 0 \\
08 \\
08 \\
0\end{array}$ & 0 \\
\hline
\end{tabular}

Fig. 2. Correlation between the choroidal thickness value ratio (between the ocular ischemic syndrome eyes and the unaffected eyes) and fluorescein angiography parameters (seconds). ACT = arm-to-choroid circulation time; ART = arm-to-retina circulation time; AVT = arteriovenous transit time.

Table 1. Baseline characteristics of patients with ocular ischemic syndrome

\begin{tabular}{lc}
\hline Characteristics & Patient $(\mathrm{n}=16)$ \\
\hline Age (yr) & $67.5(34-81)$ \\
Sex (male : female) & $15(93.8): 1(6.3)$ \\
Eye (right : left) & $7(43.8): 9(56.3)$ \\
Hypertension & $12(75.0)$ \\
Diabetes & $7(43.8)$ \\
Hyperlipidemia & $2(12.5)$ \\
Initial neovascular glaucoma & $13(81.3)$ \\
Phakia : pseudophakia & $10(62.5): 6(37.5)$ \\
Duration of symptoms (day) & $4(0-730)$ \\
\hline
\end{tabular}

Values are presented as median (range) or number (\%).

male. Seven and nine patients had OIS in the right and left eyes, respectively. Of the study subjects, 12 had hypertension, seven had diabetes and two had dyslipidemia. Thirteen patients had neovascular glaucoma at the initial presentation, and six patients had previously undergone cataract surgery. The median duration of visual symptoms was 4 days.

The ophthalmic characteristics of the OIS and the unaffected eyes are presented in Table 2. In the OIS eyes, the mean BCVA was $1.01 \pm 1.00 \log$ MAR at the initial presentation, $1.06 \pm 1.03 \log$ MAR at 1 month follow-up, and 1.33 $\pm 1.50 \log$ MAR at 6 months follow-up. On the other hand, in the OIS eyes, the mean IOP was $21.69 \pm 8.90 \mathrm{mmHg}$ in the first visit, $19.88 \pm 7.07 \mathrm{mmHg}$ at 1 month follow-up, and $18.88 \pm 7.08 \mathrm{mmHg}$ at 6 months follow-up.

BCVA and IOP were significantly different between the OIS and unaffected eyes in all the examinations. BCVA worsened over time. All the FAG parameters were delayed at the initial presentation with ACT being $25.66 \pm 7.17$ seconds, ART being $25.73 \pm 6.36$ seconds and AVT being $43.41 \pm 17.44$ seconds.

Carotid artery stenosis was evaluated by a carotid Doppler examination or a brain MRA. Twelve patients underwent carotid Doppler examination and four patients underwent brain MRA to evaluate carotid artery stenosis or occlusion. Patients whose stenosis or occlusion was unclear were three. Four had mild stenosis, one had moderate stenosis, and eight had severe stenosis.

\section{Comparisons and correlations of choroidal thickness}

We compared the choroidal thickness between the eyes with OIS and the unaffected eyes using SS-OCT in each ETDRS grid (Table 3). While the choroidal thickness of the OIS eyes in every nine areas was lesser than that of the fellow eyes, the choroidal thickness of inner temporal, outer nasal, and outer temporal area had significant differences between OIS and unaffected eye (inner temporal, 195.12 \pm 65.69 and $241.12 \pm 105.38 \mu \mathrm{m}, p=0.034$; outer nasal, 
Table 2. Comparison of clinical characteristics between OIS and fellow eyes

\begin{tabular}{lrrr}
\hline Characteristics & OIS eyes & Fellow eyes & $p$-value \\
\hline Initial BCVA (logMAR) & $1.01 \pm 1.00$ & $0.07 \pm 0.14$ & 0.001 \\
Initial IOP (mmHg) & $21.69 \pm 8.90$ & $15.06 \pm 4.42$ & 0.028 \\
1-month BCVA (logMAR) & $1.06 \pm 1.03$ & $0.29 \pm 0.45$ & 0.002 \\
1-month IOP (mmHg) & $19.88 \pm 7.07$ & $15.56 \pm 3.10$ & $0.25 \pm 0.40$ \\
6-month BCVA (logMAR) & $1.33 \pm 1.50$ & $14.31 \pm 4.54$ & 0.002 \\
6-month IOP (mmHg) & $18.88 \pm 7.08$ & & 0.023 \\
Fluorescein angiography parameters (sec) & & & \\
$\quad$ Arm-to-choroid circulation time & $25.66 \pm 7.17$ & $25.73 \pm 6.36$ & \\
$\quad$ Arm-to-retina circulation time & $43.41 \pm 17.44$ & \\
\multicolumn{1}{c}{ Arteriovenous transit time } & & & \\
\hline
\end{tabular}

Values are presented as mean \pm standard deviation.

$\mathrm{OIS}=$ ocular ischemic syndrome; BCVA = best-corrected visual acuity; logMAR = logarithm of the minimum angle of resolution; IOP $=$ intraocular pressure.

Table 3. Comparison of choroidal thickness between OIS and unaffected eyes

\begin{tabular}{lcccc}
\hline Choroidal thickness $(\mu \mathrm{m})$ & OIS eyes & Unaffected eyes & $p$-value & OIS to unaffected eyes ratio \\
\hline Central choroidal thickness & $213.19 \pm 70.39$ & $247.25 \pm 116.07$ & 0.109 & $0.96 \pm 0.33$ \\
$\quad$ Inner ring area & & & \\
$\quad$ Superior & $204.50 \pm 69.04$ & $219.00 \pm 76.72$ & 0.289 & $0.99 \pm 0.32$ \\
$\quad$ Nasal & $190.75 \pm 76.52$ & $221.94 \pm 112.26$ & 0.109 & $0.93 \pm 0.34$ \\
$\quad 184.50 \pm 62.98$ & $203.81 \pm 82.87$ & 0.179 & $0.95 \pm 0.31$ \\
$\quad$ Inferior & $195.12 \pm 65.69$ & $241.12 \pm 105.38$ & 0.034 & $0.88 \pm 0.29$ \\
$\quad$ Temporal & & & 0.842 & $0.98 \pm 0.25$ \\
$\quad$ Outer ring area & $209.19 \pm 80.82$ & $214.81 \pm 64.10$ & 0.93 & $0.97 \pm 0.56$ \\
$\quad$ Superior & $140.56 \pm 69.64$ & $159.81 \pm 79.20$ & 0.034 & $0.89 \pm 0.30$ \\
$\quad$ Nasal & $155.69 \pm 62.94$ & $188.88 \pm 87.30$ & 0.059 & $0.89 \pm 0.27$ \\
$\quad$ Inferior & $183.69 \pm 59.83$ & $215.87 \pm 73.37$ & 0.023 & $0.94 \pm 0.28$ \\
$\quad$ Temporal & $197.61 \pm 65.86$ & $226.63 \pm 93.86$ & 0.049 & $0.94 \pm 0.27$ \\
Central/inner ring area, mean & $186.35 \pm 62.60$ & $212.50 \pm 81.61$ & 0.063 & \\
Central/inner/outer ring area, mean & & & & \\
\hline
\end{tabular}

Values are presented as mean \pm standard deviation.

OIS $=$ ocular ischemic syndrome.

$140.56 \pm 69.64$ and $159.81 \pm 79.20 \mu \mathrm{m}, p=0.034$; outer temporal, $183.69 \pm 59.83$ and $215.87 \pm 73.37 \mu \mathrm{m}, p=0.049$, respectively). The mean total thickness of center and inner ring area was significantly thinner in the OIS eyes compared to the unaffected eyes $(197.61 \pm 65.86$ and 226.63 $\pm 93.86 \mu \mathrm{m}, p=0.049$, respectively). The correlation between the ratio of OIS to unaffected eyes and FAG param- eters is illustrated in Fig. 2, and there were no statistically significant differences noted. Comparisons of thickness in retina, retinal nerve fiber layer, and ganglion cell layer between OIS eyes and unaffected eyes are presented in Supplementary Table 1.

We also conducted follow-up OCT in OIS eyes and compared the choroidal thickness between the initial examina- 
Table 4. Comparison of choroidal thickness between initial and follow-up ocular ischemic syndrome eyes

\begin{tabular}{|c|c|c|c|c|}
\hline Choroidal thickness $(\mu \mathrm{m})$ & Initial & Follow-up & $p$-value & Changes ${ }^{*}$ \\
\hline Central choroidal thickness & $213.19 \pm 70.39$ & $202.50 \pm 73.39$ & 0.363 & $10.69 \pm 46.92$ \\
\hline \multicolumn{5}{|l|}{ Inner ring area } \\
\hline Superior & $204.50 \pm 69.04$ & $201.13 \pm 72.09$ & 0.979 & $3.38 \pm 43.51$ \\
\hline Nasal & $190.75 \pm 76.52$ & $192.38 \pm 85.82$ & 0.918 & $-1.63 \pm 29.58$ \\
\hline Inferior & $184.50 \pm 62.98$ & $198.62 \pm 75.47$ & 0.125 & $-14.13 \pm 35.45$ \\
\hline Temporal & $195.12 \pm 65.69$ & $192.81 \pm 70.12$ & 0.712 & $2.31 \pm 44.14$ \\
\hline \multicolumn{5}{|l|}{ Outer ring area } \\
\hline Superior & $209.19 \pm 80.82$ & $207.00 \pm 92.91$ & 0.856 & $2.19 \pm 59.80$ \\
\hline Nasal & $140.56 \pm 69.64$ & $149.06 \pm 71.78$ & 0.326 & $-8.50 \pm 43.43$ \\
\hline Inferior & $155.69 \pm 62.94$ & $181.38 \pm 63.80$ & 0.066 & $-25.69 \pm 57.04$ \\
\hline Temporal & $183.69 \pm 59.83$ & $189.31 \pm 69.46$ & 0.423 & $-5.62 \pm 30.09$ \\
\hline Central/inner ring area, mean & $197.61 \pm 65.86$ & $197.49 \pm 70.94$ & 0.877 & $0.13 \pm 32.99$ \\
\hline Central/inner/outer ring area, mean & $186.35 \pm 62.60$ & $190.47 \pm 68.05$ & 0.496 & $-4.11 \pm 29.44$ \\
\hline
\end{tabular}

Values are presented as mean \pm standard deviation.

*Initial follow-up.

\begin{tabular}{|c|c|c|c|c|c|c|c|c|c|}
\hline $\begin{array}{c}0 \\
0 \\
0 \\
800 \\
80^{\circ}\end{array}$ & 0 & $0^{0}{ }^{0} 80^{8} 0^{8}$ & $\begin{array}{c}0 \\
0^{\circ} \circ \\
000 \\
000 \\
00\end{array}$ & $\begin{array}{l}0 \\
0 \\
0 \\
0 \\
0 \\
0 \\
0 \\
0\end{array}$ & $\begin{array}{l}\circ \\
\circ 0 \\
\circ 8 \\
080 \\
08\end{array}$ & 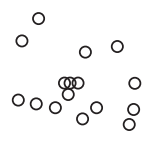 & $\begin{array}{r}0 \\
80 \\
0.80 \\
0.80 \\
0 \quad 0\end{array}$ & $\begin{array}{c}0 \\
0 \\
0 \\
0 \\
0 \\
0\end{array}$ & 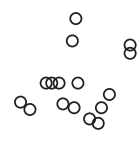 \\
\hline $\begin{array}{l}\circ .0 \\
0 \\
0.0800 \\
0 \\
0\end{array}$ & 0 & $0^{\circ}{ }_{0}^{\infty}{ }_{0}^{0}$ & $\begin{array}{c}0 \\
0 \\
0.0080 \\
0\end{array}$ & $\begin{array}{c}0 \\
0 \\
0 \\
0 \\
\substack{\alpha_{0} \\
0} \\
c\end{array}$ & $\begin{array}{ll}0 & \\
0 & 0 \\
0800 & 0 \\
8 & 0\end{array}$ & $\begin{array}{lll}0^{\circ} & 0 \\
0 & \infty & 8 \\
0 & 8\end{array}$ & $\begin{array}{c}8 . \\
\infty 8_{8}^{8}\end{array}$ & $\begin{array}{r}0 . \\
0 \text { 。 } \\
0\end{array}$ & 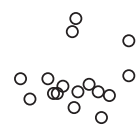 \\
\hline $\begin{array}{c}0 \\
\circ \\
800 \\
80 \\
80\end{array}$ & $\circ$ & $\begin{array}{c}0 \\
0 \\
0 \\
8^{8} \circ \\
8^{\circ} 8\end{array}$ & $\begin{array}{c}0 \\
0 \\
0 \\
0 \\
0 \\
g_{0}^{\infty}\end{array}$ & $\begin{array}{l}0 \\
0_{0}^{8} \\
8_{\infty}^{8}\end{array}$ & $\begin{array}{l}\circ . \\
880 \\
88^{\circ} \\
0 \\
0\end{array}$ & 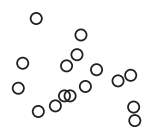 & $\begin{array}{c}0 \\
0 \\
080 \\
0 \\
80 \\
0 \\
0\end{array}$ & $\begin{array}{r}0 \\
0 \\
8 \\
8 \\
8 \\
0 \\
0 \\
0 \\
0 \\
0\end{array}$ & ${ }_{0}^{\circ} \infty_{0}^{\infty} 8$ \\
\hline
\end{tabular}

Fig. 3. Correlation between changes in the choroidal thickness of cular ischemic syndrome eyes during follow-up (initial follow-up, $\mu \mathrm{m}$ ) and fluorescein angiography parameters (seconds). ACT = arm-to-choroid circulation time; ART = arm-to-retina circulation time; AVT = arteriovenous transit time.

tion and follow-up examination (Table 4). The median duration from the initial to follow-up OCT was 39 days (range, 14-210). However, there was no statistically significant change in choroidal thickness in the OIS eyes during follow-up in any of the nine areas. We also evaluated the correlation between the amount of changes in choroidal thickness in the OIS eyes during follow-up and FAG parameters (Fig. 3), but no significant associations were revealed. 


\section{Discussion}

As choroid is an irregular structure with uneven contour, it is hard to evaluate the overall changes in choroid. The previous study investigated choroidal volume changes in the ETDRS area to evaluate the choroidal change [10]. Whereas the choroidal thickness in every nine areas of ETDRS was investigated in this study. We evaluated the clinical significance of choroidal thickness in the OIS affected eye and found that the overall choroidal thickness of the eyes with OIS was lower than that of the unaffected eyes. But there were significant differences in only limited areas of the ETDRS grid. In addition, we could not find further changes in the choroidal thickness between the initial and follow-up examinations, and there was no correlation between choroidal thickness and FAG parameters.

Decreased visual acuity is the most common symptom at the initial visit in patients with OIS and is observed in more than $90 \%$ of cases [3]. In the present study, the OIS eye presented a lower BCVA and higher IOP than the unaffected eyes at the initial examination. Thus increased IOP may have been influenced by the patients with neovascular glaucoma. Subsequently, BCVA was worse, and IOP improved with serial follow-up at 1 and 6 months, but the difference in comparison to the unaffected eye still existed.

Overall, the choroidal thickness was lesser than that of the unaffected eye, but the difference was statistically significant only in the inner ring area, including the center. This is thought to be because the subfoveal choroid, which is structurally the thickest in the entire choroid, is more likely to be affected by OIS. It is well known as the thickest choroid located subfoveal, with peripheral thinning, in normal eyes [6].

Decreased choroidal circulation due to carotid artery stenosis may affect the choroidal thickness. Kim et al. [10] demonstrated that unilateral OIS eyes have a thinner subfoveal choroid than unaffected eyes. They interpreted that this indicates impaired choroidal circulation in patients with OIS, but they did not evaluate choroidal and retinal changes in the OIS eyes during serial evaluations. Kang et al. [9], assumed that OIS could be the end-stage presentation in the gradual course of ischemic cerebrovascular disease and choroidal thinning might begin to develop before onset of OIS. In this study, we evaluated changes in retinal thickness and choroidal thickness in both OIS and unaf- fected eyes, and the time-dependent changes in choroidal thickness were compared. These changes in choroidal thickness were observed to be insignificant in the current study. We inferred that the choroidal structure may have been damaged in the early stages of OIS, so that only minor choroidal thickness changes occurred in the OIS eyes during the follow-up period.

Prolonged FAG parameters are common and important signs in the diagnosis of OIS. Prolonged ART is the most specific, but not the most sensitive, FAG sign of OIS. The most sensitive angiographic sign is prolonged AVT, but it is not OIS-specific [11]. In this study, all the FAG parameters including ACT, ART, and AVT had a time delay, which is consistent with a previous study [12]. We also investigated the correlation between FAG parameters and choroidal thickness. The choroidal thickness value ratio (between the OIS eyes and the unaffected eyes) and the change in choroidal thickness in the OIS eyes during follow-up were used in the analysis. No statistically significant correlations were found in the current study. Kim et al. [12] reported that there were no significant differences in the parameters of FAG between OIS patients with and without neovascular glaucoma. Therefore, it seems difficult to describe the FAG parameters as a single independent factor in eyes with OIS. We believe this is because the circulation of the choroid is mainly affected by posterior ciliary artery, and it is less accurately analyzed by FAG examination. To our knowledge, this study is the first to analyze the association between choroidal thickness and FAG parameters in OIS affected eye and to evaluate the clinical significance of choroidal thickness with regard to serial examinations in patients with OIS.

There are some limitations to this study, including its retrospective nature and small sample size. These factors could result in selection bias and affect outcomes. We could not evaluate carotid artery stenosis with a single method and used carotid Doppler test or MRA, but $81 \%$ patient had carotid artery stenosis. Carotid Doppler examination allows detection of carotid artery stenosis not in $100 \%$, but at least $75 \%$ of cases [3]. In this study, we decided to diagnose these patients as OIS based on obvious findings in clinical symptoms, slit-lamp microscopy and fluorescein angiography without confirming of the carotid Doppler examination or brain MRA. The current study only covers the short follow period. Although we considered that changes in the choroidal vascular structure had 
already occurred at the time of diagnose, it would have been better if the longer follow-up results were reinforced. Further evaluations will be needed. Although the measurement of choroidal thickness was conducted by skilled specialists, human-based errors and biases cannot be completely ruled out. Nevertheless, the present study contributes important information to the field of ophthalmology and suggests directions for further research on choroidal thickness and OIS.

In conclusion, we found that the choroidal thickness of the eyes with OIS was significantly less than that of the unaffected fellow eyes, especially in the central area of the macula. It can be stated that this reduction of choroidal thickness takes place in the early phase of the disease, which is substantiated by the fact that no significant changes in choroidal thickness were noted during the follow-up period. Further large-scale and prospective studies are needed to verify the clinical significance of choroidal thickness in eyes with OIS.

\section{Conflicts of Interest}

No potential conflict of interest relevant to this article was reported.

\section{Acknowledgements}

None.

\section{Funding}

The authors received no financial support for this article.

\section{Supplementary Materials}

Supplementary Table 1 is available from: https://doi. org/10.3341/kjo.2021.0155.

\section{References}

1. Mendrinos E, Machinis TG, Pournaras CJ. Ocular ischemic syndrome. Surv Ophthalmol 2010;55:2-34.

2. Young LH, Appen RE. Ischemic oculopathy. A manifestation of carotid artery disease. Arch Neurol 1981;38:358-6.

3. Terelak-Borys B, Skonieczna K, Grabska-Liberek I. Ocular ischemic syndrome: a systematic review. Med Sci Monit 2012;18:RA138-44.

4. Sivalingam A, Brown GC, Magargal LE, Menduke H. The ocular ischemic syndrome. II. Mortality and systemic morbidity. Int Ophthalmol 1989;13:187-91.

5. Malhotra R, Gregory-Evans K. Management of ocular ischaemic syndrome. Br J Ophthalmol 2000;84:1428-31.

6. Manjunath V, Goren J, Fujimoto JG, Duker JS. Analysis of choroidal thickness in age-related macular degeneration using spectral-domain optical coherence tomography. $\mathrm{Am} \mathrm{J}$ Ophthalmol 2011;152:663-8.

7. Nakayama M, Keino H, Okada AA, et al. Enhanced depth imaging optical coherence tomography of the choroid in Vogt-Koyanagi-Harada disease. Retina 2012;32:2061-9.

8. Spaide RF, Koizumi H, Pozzoni MC. Enhanced depth imaging spectral-domain optical coherence tomography. Am J Ophthalmol 2008;146:496-500.

9. Kang HM, Choi JH, Koh HJ, Lee SC. Significant changes of the choroid in patients with ocular ischemic syndrome and symptomatic carotid artery stenosis. PLoS One 2019;14:e224210.

10. Kim DY, Joe SG, Lee JY, et al. Choroidal thickness in eyes with unilateral ocular ischemic syndrome. J Ophthalmol 2015;2015:620372.

11. Brown GC, Magargal LE. The ocular ischemic syndrome. Clinical, fluorescein angiographic and carotid angiographic features. Int Ophthalmol 1988;11:239-51.

12. Kim YH, Sung MS, Park SW. Clinical features of ocular ischemic syndrome and risk factors for neovascular glaucoma. Korean J Ophthalmol 2017;31:343-50. 


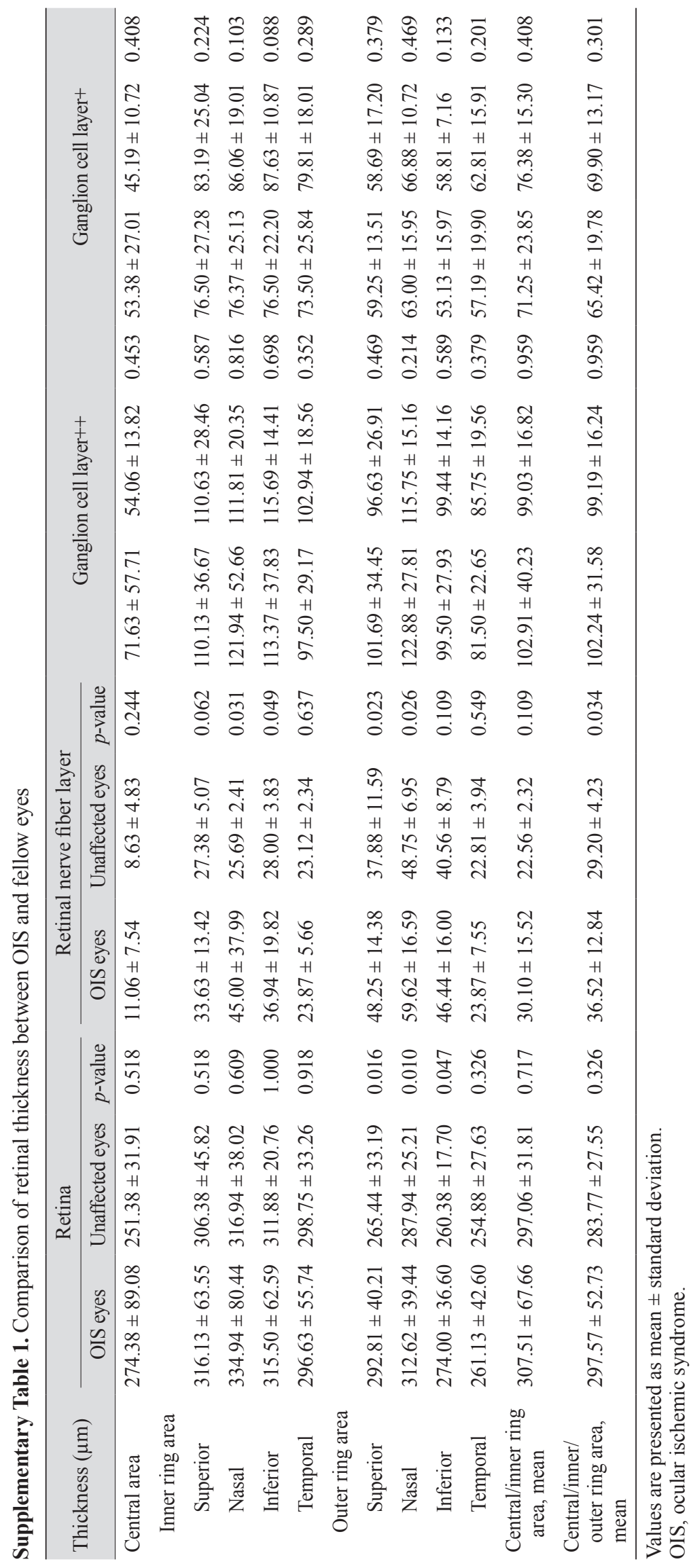

